\title{
"Il y a des mamans qui aiment le travail et des mamans qui aiment jouer » Reformulation orale, atelier d'écriture et compétences en littératie
}

\author{
HÉLÈNE CASTANY-OWHADI \\ Université de Montpellier
}

YVES SOULÉ

Université de Montpellier

MARTINE DREYFUS

Université de Montpellier

\section{Résumé}

La présente étude explore le rôle des reformulations orales au sein d'un atelier d'écriture en première année du primaire, dans une visée compréhensive de l'activité conjointe ${ }^{1}$ pour le développement des compétences en littératie. À partir d'un extrait de verbatim dans une classe particulièrement performante, nous étudions les jeux de reformulations entre l'enseignant et un élève en insécurité scripturale en mettant en évidence la complexité du processus d’étayage.

\section{Mots-clés}

Écriture, enseignement-apprentissage, école primaire, reformulation orale, littératie

\section{Introduction}

Cet article s'appuie sur une thèse en cours qui étudie l'oral dans une perspective didactique à partir de l'étude de la reformulation, considérée comme une ressource langagière déterminante dans et pour l'action didactique. Il s'inscrit dans la continuité des travaux initiés par la DFLM ${ }^{2}$ et des chercheurs de Montpellier sur «l'écrit et l'oral réflexif », dans laquelle la reformulation est appréhendée comme un "mécanisme central de la réflexivité » (Chabanne \& Bucheton, 2002). Elle est étudiée dans le contexte de séances d'atelier de production écrite au CP, Cours Préparatoire (première année de primaire au Québec), données produites par la recherche LireÉcrire $C P^{3}$ de l'Institut français de l'éducation (IFé) intitulée «Étude de l'influence des choix didactiques des enseignants sur la qualité des apprentissages des élèves en lecture et en écriture entre 5 et 8 ans » (2012-2015).

\footnotetext{
${ }^{1}$ Au sens de Sensevy (2011) qui, dans sa théorie de l'action conjointe en didactique, affirme que l'on ne peut comprendre l'action de l'élève sans faire le lien avec l'action de l'enseignant dans le savoir.

2 Didactique du Français Langue Maternelle

${ }^{3}$ Recherche coordonnée par R. Goigoux et dont la finalité est d'identifier les caractéristiques des pratiques pédagogiques ordinaires qui s’avèrent efficaces et équitables mais aussi de produire des connaissances scientifiques nouvelles sur les caractéristiques de ces pratiques. Pour une première synthèse des résultats de cette recherche voir le Rapport de recherche, Lire et Ecrire , 2016. En ligne sur le site de l’Institut français de l’éducation (Ifé) : <http://ife.ens-lyon.fr/ife/recherche/lireecrire>.
} 
Les résultats de cette recherche (Goigoux, 2016) nous ont conduits à approfondir le rôle des reformulations orales du maitre dans une visée compréhensive de l'activité de l'enseignant pour l'apprentissage du lire-écrire-parler (Castany-Owhadi, Soulé \& Dreyfus, soumis ; Dreyfus, Soulé, Dupuy \& Castany-Owhadi, 2017). Dans cet article, à partir d'un extrait de verbatim d'une classe particulièrement performante du corpus (rang 2 pour l'ensemble des performances évaluées, rang 8 pour les performances réalisées en écriture sur 131 classes), nous essayons de montrer en quoi les reformulations donnent à voir ce qui caractérise la littératie c'est-à-dire l'interaction dans le processus d'enseignementapprentissage du lire-écrire-parler, mais aussi le rôle de celles-ci dans le processus d'étayage.

\section{Littératie et compétence en littératie}

\section{Problématique et objectifs}

Le concept de littératie ne fait pas consensus dans la communauté des chercheurs francophones (Hébert \& Lépine, 2013). Dans son sens restreint, il renvoie au savoir lireécrire, mais dans son acception plus extensive et notamment pour l'équipe ÉRLI ${ }^{4}$, il englobe aussi l'oral du fait de « l'interdépendance étroite existant dans l'apprentissage du lire-écrire et parler » (Hébert \& Lafontaine, 2010, p.5). Ces auteures affirment d'ailleurs que l'intérêt de ce concept est précisément de mettre en lumière cette interdépendance, Hébert (2010) montrant en outre qu’il est nécessaire de favoriser les approches intégratrices de la lecture, de l'écriture et de l'oral, un des enjeux majeurs de la scolarisation résidant dans le développement des habiletés associées à la littératie (Hébert \& Lépine, 2013).

Dans le cadre de cet article, nous retenons cette conception intégratrice de la littératie. Elle fait écho au «modèle culturel du sujet écrivant » (Bucheton, 2014) qui combine des activités prenant en compte « le dire - lire - écrire - parler - regarder » (p.220) ainsi que le développement de la personne, comme dans la conception d'Hébert et Lépine (2013) : «La littératie touche d'une part un ensemble d'attitudes, de connaissances, d'habiletés et de compétences qui sont, d'autre part, à mesurer ou à situer dans une dynamique espace/temps et dans une visée émancipatrice du développement de la personne » (p.25). Nous retenons aussi la définition de « compétence en littératie » comme « capacités d'utiliser le langage et les images de formes riches et variées pour voir, écouter, parler, lire, écrire, comprendre, communiquer, penser de façon critique afin d'atteindre un but et de développer ses connaissances en classe, à l'école, à la maison ou dans la communauté » (Moreau, Leclerc \& Landry, 2010, p. 43).

\section{De la difficulté et de l'intérêt à entrer dans le langage écrit}

L'entrée dans l'écrit constitue un apprentissage difficile pour un enfant de première année de primaire et l'école joue sur ce point un rôle fondamental, étant indissociablement liée à l'écriture comme instrument de la culture écrite (Goody,1987).

Pourtant, sans nier cette complexité, de nombreux travaux notamment sur les écritures inventées en maternelle ( invented spelling ») ont montré l'intérêt d'une pratique précoce : si Chomsky (1971) défendait déjà l'idée qu'il faut écrire avant d’apprendre à lire, à la suite de cette auteure, Ferreiro et Gómez Palacio (1988), étudiant la psychogenèse de l'écrit, ont établi que les enfants conceptualisent la langue écrite avant son apprentissage ; Ehri (1989), Rieben, Ntamakiliro, Gonthier et Fayol (2005) et Sénéchal, Ouellette, Pagan

\footnotetext{
${ }^{4}$ Équipe de Recherche en Littératie et Inclusion dirigée par L. Lafontaine (UQO).
} 
et Lever (2012) affirment que l'apprentissage de l'orthographe («spelling ») renforce l'apprentissage de la lecture et de l'orthographe. Rieben (2003) affirme quant à elle « qu'une façon indirecte d'évaluer la pertinence de la pratique des écritures inventées pour l'apprentissage de la lecture peut (...) consister à s’interroger sur les liens qu'elles entretiennent avec la conscience phonologique et la connaissance des lettres » (p.31).

En France, après que Besse (2000) a identifié des préoccupations visuographiques, phonographiques et orthographiques chez les apprentis scripteurs de maternelle, les travaux de Jaffré (2003) et David (2003) ont souligné l'intérêt des " commentaires métagraphiques » permettant à l'enfant d'expliciter la raison d'être de ses productions graphiques. Morin et Montésinos-Gelet (2007) ont ensuite montré que la pratique des « écritures approchées » autorise le développement des habiletés littéraciques.

S’agissant du $\mathrm{CP}^{5}$, soit pour justifier la pratique de l'écriture dès le début d'année (Garcia-Debanc, 2003), soit dans le cadre de l' " atelier dirigé d'écriture " ${ }^{6}$ centré sur l'agir enseignant et les gestes professionnels ${ }^{7}$ (Bucheton \& Soulé, 2009a), soit dans le cadre de l'analyse des interactions verbales en contexte de production écrite (Mauroux, David \& Garcia-Debanc, 2015) les conclusions sont identiques qui revendiquent l'interaction du lire-écrire-parler.

De plus, des travaux récents souhaitent contribuer au débat sur les relations entre pratiques enseignantes et apprentissages des élèves en lecture-écriture au début de l'école élémentaire (Goigoux, Jarlégan \& Piquée, 2015 ; Totereau \& Dreyfus, 2017).

\section{Reformulation et étayage}

Dès lors que l'on s'essaie à la production écrite en classe, l'activité dialogale est nécessaire pour les scripteurs novices (Bucheton \& Soulé, 2009a) et le rôle de l'enseignant déterminant notamment pour les élèves qui n’ont pas un "rapport-scriptural scolaire au langage » (Lahire, 2008). D’où l'importance du concept d'étayage que l'on doit à Bruner (1983), et du lien que nous posons avec la reformulation. La traduction du terme anglais « scaffolding » qui signifie littéralement " échafaudage », montre bien l'aide temporaire apportée par l'enseignant à l'élève dans sa zone proximale de développement. Cependant, comme le souligne Nonnon (1990, p. 156), « l'analyse que fait Bruner de l'interaction de tutelle et des fonctions d'étayage de l'activité de l'enfant assumées par l'adulte (...) ne passe pas par une analyse précise des modalités verbales de l'échange ». Or cette analyse nous parait essentielle. D’une part, comme le montre Hudelot (1993), les conduites d'étayage langagier sont à étudier en fonction des effets sur l'interlocuteur enfantin ; d'autre part elles rendent compte de la dynamique des postures ${ }^{8}$ d'étayage telles qu'elles ont été travaillées dans un contexte d' " ateliers dirigés d'écriture » en première année de primaire (Bucheton \& Soulé, 2009a).

\section{Question et objectifs de recherche}

\footnotetext{
${ }^{5}$ Cours Préparatoire (première année du primaire en France).

${ }^{6}$ Au sens d'atelier de production d'écrits en petit groupe avec présence de l'enseignant-e.

7 « Les mots, les attitudes, les comportements qui déterminent la manière dont un enseignant conduit une séance » (Bucheton \& Soulé, 2009a, p. 26).

8 «Les « postures » sont des schèmes d’actions cognitives et langagières, disponibles et préformées, que le sujet convoque en réponse à une situation rencontrée ou une tâche scolaire donnée » (Bucheton, 2014, p.101), autrement dit des « modes de penser-parler-lire-écrire-agir » (ibid.).
} 
Notre question de recherche peut être formulée de la manière suivante : peut-on considérer les reformulations du maitre et des élèves comme un phénomène langagier favorisant le développement des compétences en littératie? Nous essayons ainsi de comprendre l'articulation du lire-écrire-parler en montrant que la reformulation orale en est une des manifestations.

\section{Cadre théorique : la reformulation}

Un " mécanisme central de la réflexivité » au service de la construction des savoirs

Dans leur ouvrage traitant de l'écrit et de l'oral réflexifs, Chabanne et Bucheton (2002) définissent la réflexivité comme «la prise de distance à l'égard de l'expérience immédiate que permet le langage » (p. 5), celle-ci impliquant une dimension épi- et métalinguistique: en effet, " tout en parlant, en écrivant, je suis dans une attitude permanente de contrôle de ce que je dis-écris, qui peut être plus ou moins intentionnelle. C'est pourquoi parler/écrire, c'est nécessairement entrer dans des normes et des contraintes linguistiques, discursives, culturelles...afin de prendre part au jeu langagier » (p. 6). La reformulation est un phénomène langagier épilinguistique (Garcia-Debanc, 2012) qui joue un rôle fondamental dans l'élaboration des connaissances, la reprise-modification (François, 1990) de la parole de l'autre contribuant à créer un espace intersubjectif commun: les auteurs défendent ainsi l’idée que la reformulation est un «mécanisme central de la réflexivité », car «la réflexivité se développe par la capacité à reprendretransformer (François, 1990) des formes discursives dans un chantier continu » (ibid., p. : 10). Jaubert et Rebière (2002) affirment aussi qu'un oral réflexif se caractérise par «le travail cognitif et langagier de «secondarisation » des pratiques langagières initiales des élèves et la construction de positions énonciatives favorisant les déplacements » (p.168), par la « mise à distance des formulations » (p.175). Dans le cadre de l'étude d'un moment de lecture-feuilleton en dernière année de maternelle, Chemla et Dreyfus (2002) montrent notamment que « les enchaînements se font le plus souvent par accrochage sur le tour de parole de l'autre, en complétant ou en modifiant l'énoncé précédent » (p. 111) autrement dit par la reformulation.

\section{Conception de la reformulation}

La reformulation est ici définie comme toute unité linguistique produite dans l'interaction orale à partir d'une unité linguistique antérieure, en contexte scolaire et plus précisément didactique: l'enseignement-apprentissage du lire-écrire-parler. Elle constitue pour nous une ressource orientée pour l'action et un geste professionnel langagier. Cette définition s’inspire de celle d'Apothéloz (2007) qui la considère comme « une répétition purement formelle d’une séquence de format quelconque », ce qui nous permet d'intégrer dans notre analyse des reformulations portant sur des unités inférieures au mot (syllabe, lettre, phonème), car ces reformulations sont importantes dans les situations de lecture et d'écriture. Nous employons le mot reformulation comme terme générique pour désigner à la fois ce qui relève de la reformulation, de la reprise et de la répétition (Rabatel, 2010).

Cette recherche s’inscrit dans la continuité des travaux qui étudient la reformulation orale en contexte didactique dans le cadre de la construction des savoirs (Brixhe \& Spécogna, 1999 ; Jaubert \& Rebière, 2001 ; Milian, 2005 ; Moussi, 2015 ; Volteau \& Garcia-Debanc, 2008). Nous prenons notamment en compte le fait qu’elle soit considérée 
comme « un élément de l'expertise professionnelle » (Garcia-Debanc, 2007) ainsi qu'un « analyseur de l'activité » (Marin, 2011), les reformulations de l'enseignant pouvant aussi jouer un rôle dans la construction des inégalités scolaires (ibid.).

Notre étude prend en compte les reformulations qui lient deux énoncés éloignés que Gülich et Kotschi (1987) nomment « reformulations différées ». La plupart des recherches sur la reformulation en contexte didactique font référence à leurs travaux notamment en utilisant les termes d' " énoncé-source » (ES) et d' " énoncé reformulateur » (ER) qui sont constitutifs de toute reformulation, l' « auto-reformulation » et l' " hétéro-reformulation » (selon qu'il s'agit de l'énoncé du même locuteur ou non) ainsi que leur modèle classificatoire. Nous utilisons celui affiné par de Gaulmyn (1987) qui distingue trois actes de reformulation : la paraphrase, la répétition et la correction.

À ce modèle classificatoire qui rend plutôt compte de l'usage linguistique et discursif de la reformulation dans l'interaction, nous avons proposé une typologie (Castany-Owhadi et al., soumis) qui prend en considération les dimensions paraverbales, non verbales ainsi que les commentaires associés aux reformulations contenant de l'écrit négocié, en cours de production ou révisé, autrement dit de l' « écrit dans l'oral » ${ }^{9}$ (Marty,1991), en inventoriant leurs fonctions didactiques et pédagogiques (voir infra p.7 et suivantes).

\section{Type d'étude}

\section{Méthodologie}

Il s’agit d'une étude exploratoire à visée descriptive (Gagné et al., 1989). Nous faisons le choix d'analyser un extrait de verbatim dans une des classes les plus performantes ${ }^{10}$ (classe 61). Nous étudions les jeux de reformulation entre l'enseignant et un élève en « insécurité scripturale » (Dabène, 1991) en mettant en évidence toute la complexité du processus d'étayage.

\section{Contexte d'étude}

L'extrait de verbatim provient de la séance 8 lors de la troisième semaine d'enquête (mai). Le CP de la classe 61 est un cours simple de 20 élèves lors de la semaine en mai avec un maitre qui a seize ans d'ancienneté dans le métier, dont six ans en première année de primaire. Celui-ci a une pratique fréquente de l'atelier dirigé pendant que les autres élèves fonctionnent en autonomie. Dans le cadre de la recherche LireÉcrireCP, un enquêteur par classe avait notamment pour mission de décrire les pratiques des enseignants ainsi que le contexte selon une méthodologie de travail définie par un cahier des charges ${ }^{11}$. L'enquêteur affirme notamment être " frappé par la pertinence (...) et par l'efficacité du maitre (...), dans un contexte difficile et une méthode d'apprentissage de la lecture qui présente de graves lacunes » (ibid.). Selon son interprétation, cette efficacité serait due à

\footnotetext{
9 Nous empruntons à Marty (1991) l'expression d' « écrit dans l’oral » pour qualifier le discours qui prend pour objet l'écrit à produire ou l'écrit produit.

10 Cf. Annexe 5.

${ }^{11}$ En ligne sur le site de l’IFé : http://ife.ens-lyon/recherche/lire-ecrire/rapport/annexe-a.3.4.1-cahier-descharges-de-lenque302teur.
} 
plusieurs causes combinées « mais peut-être surtout des reformulations (et non répétitions) très fréquentes, mais trop brèves pour être notées comme tâches spécifiques » (ibid.). ${ }^{12}$

\section{L'extrait : les données produites}

La séance 8 (66 minutes) comporte deux ateliers de production d'écrits en petit groupe avec au début, une phase de définition et d'organisation de l'écrit à produire de 20 minutes. Le support d'écriture est le début d'une phrase extraite d'un album Le livre des mamans $^{13}$ (Todd Parr). Après un rappel oral du récit avec les enfants, le maitre écrit au tableau blanc interactif (TBI) «Il y a des mamans qui... », et il demande aux élèves : « Alors qu'est-ce qu'on pourrait écrire comme suite ? ». Il recueille à l'oral les propositions des enfants et celles-ci sont discutées par l'ensemble des élèves, ensuite une partie des élèves réalise un travail de lecture-compréhension en autonomie alors que l'enseignant prend avec lui un petit groupe d'enfants qu'il guide dans le travail d'écriture. Lors de cette séance, il y a deux ateliers de production d'écrits qui se font de manière successive sachant qu'un enseignant surnuméraire participe aux ateliers « tournants » en prenant deux fois un petit groupe d'élèves en dehors de la classe.

L'extrait choisi (annexe 1), d'une durée de 5 minutes, provient de cette séance à la $43^{\mathrm{e}}$ minute, au début du deuxième atelier d'écriture de 5 élèves. En début d'année, l'enquêteur a notamment demandé à l'enseignant de choisir un élève (B) « dont l'avenir en lecture et en écriture est jugé, au début du CP, inquiétant par l'enseignant au sens où il ne sait pas si l'élève parviendra à réaliser les apprentissages attendus à la fin du cours préparatoire » (rapport LireÉcrireCP, p. 192) ${ }^{14}$. Le verbatim transcrit des échanges avec l'élève $\mathrm{B}$ lors de la phase de mise en texte, le maitre apportant un étayage complexe pour aider l'élève à mettre en mots la première phrase qu'il souhaite écrire : « Il y a des mamans qui aiment le travail et des mamans qui aiment jouer. »

\section{Méthode d'analyse}

Nous utilisons le modèle classificatoire de Gülich et Kotschi (1987) repris par De Gaulmyn qui distingue trois actes de reformulation :

- la paraphrase

Elle se définit comme une reformulation avec une relation sémantique entre l'énoncésource (ES) et l’énoncé reformulateur (ER).

29. M : qu'est'-ce qu'on pourrait écrire [ES],+/ allez+ est'-ce qu'on (ne) pourrait pas se donner des idées un petit peu ensemble (mouvement des bras) de façon à avoir voilà + quelques idées de ce qu'on pourrait écrire $[E R](\ldots)$ /

(Classe 61, séance 8, mai)

\footnotetext{
${ }^{12}$ Cet avis, facultatif, est proposé à la fin des trois semaines d’enquête avant le traitement des données. Il n’a pas de valeur scientifique en soi, mais il nous intéresse parce qu'il pointe l'importance des reformulations.

13 Annexe 4.

14 Les enquêteurs ont notamment photographié et déposé sur le site web de l’IFé dédié à la recherche LireÉcrireCP les traces d'écriture de l'élève B (Annexe 3 pour la semaine en mai) lors des trois semaines d'observation.
} 
- la répétition 15

Elle se caractérise par les mêmes mots qui réapparaissent, mais il peut y avoir abrègement, expansion ou réarrangement (de Gaulmyn, 1987). Ici, l’équivalence sémantique est maximale entre l'énoncé-source et l'énoncé reformulateur.

308. M : (à une élève) hélicoptèrE [ES]+ hélicoptèrE, [ER]+ /

(Classe 61, séance 8, mai)

- la correction

Il y a une relation sémantique de «contraste » (Gülich \& Kotschi, 1987) avec une modification d’un élément de l’énoncé-source considéré comme « fautif ».

53.E1 : il y a des mamans i(ls) travaillent à la mairie, [ES]+ /

54.M : il y a des mamans QUI travaillent à la mairie, [ER]+ /

Nous nous référons aussi aux catégories de « reformulations multi-modales ${ }^{16}$ » à des fins didactiques (Castany-Owhadi et al., soumis) que nous illustrons à partir d'exemples de la classe $23^{17}$, l'énoncé-source se situant au tour de parole 102 (E : « sinon le loup va te dévorer ») :

- la reformulation oralo-graphique : c’est une répétition d’un énoncé-source de l' "écrit dans l'oral » par la relecture de l'écrit produit.

Ex : 318.M : (...) (en relisant l'écrit d’A.) le loup va te dévorer,+/ (...)

- la reformulation gestuo-oralo-graphique : la répétition-relecture de l'écrit produit est associée à un geste de pointage des mots par l'enseignant ou par l'élève.

Ex : 264.M : (en relisant l'écrit de L. avec pointage du doigt) le loup va (L. écrit),+/

- la reformulation prosodique : cette répétition se caractérise par une accentuation sur un phonème, une syllabe ou un mot. Elle peut aussi se distinguer de l'énoncé-source par une pause entre les phonèmes, les syllabes ou les mots, mais aussi par une variation de l'intonation.

Ex : 231.M : (...) le loup V:::a,+/

- la reformulation prosodico-gestuelle : elle se distingue par une pause importante entre les

15 Nous utilisons aussi les «patterns de reformulation » d’Apothéloz (2005) pour distinguer les types de répétition : répétition simple, répétition avec substitution, répétition avec insertion, répétition incomplète à droite et répétition avec continuation.

16 « multi-modales » au sens de Rabatel (2010) : ce qui caractérise le langage oral, « avec ses éléments proprement linguistiques, ses paramètres voco-mimo-posturo-gestuels paraverbaux auxquels s'ajoutent des éléments proxémiques » (p.8).

17 Séance 14 en novembre où les élèves sont amenés à écrire « Le loup va te dévorer. ». 
syllabes ou les mots avec un geste de démarcation avec les mains.

Ex : 203.M : (...) (avec geste de démarcation avec les mains) le++ loup+++ va (...)

/

- la reformulation phono-gestuelle : nous pouvons la définir comme une répétition avec accentuation sur un phonème accompagné d’un geste des mains.

Ex : 342. M : (avec geste Borel-Maisonny $\left.{ }^{18}\right)[\boldsymbol{d}]+[\boldsymbol{d}]+[\boldsymbol{d}],+/$

- la reformulation gestuelle : un geste accompagne une répétition de l’ « écrit dans l’oral ».

À ces «reformulations multi-modales ", nous avons aussi distingué des reformulations associées à un commentaire sur l' « écrit dans l'oral » c'est-à-dire l'écrit négocié, produit ou révisé.

$\mathrm{Ex}: 349: \mathrm{M}:(\ldots)$ tu sais quoi'+/ ce soir on va dire à maman que $\mathbf{t}(\mathbf{u})$ as écrit la phrase+ le loup va te dévorer tout seul,+/

Nous utilisons aussi le «modèle des postures d’étayage » (Bucheton \& Soulé, 2009b) en nous référant à trois postures dans notre analyse :

- la «posture d'accompagnement » : le maitre évite notamment de donner la réponse en se retenant d'intervenir.

- la « posture d'enseignement » : le maitre nomme les savoirs.

- la « posture réflexive » : l'élève est dans l'agir, mais aussi dans la réflexion sur son agir.

Les auteurs montrent notamment que lorsque l'enseignant est en "posture d’accompagnement » dominante, les élèves sont en « posture réflexive ».

\section{Analyse de l'extrait ${ }^{19}$}

L'extrait commence au tour de parole 374. Le maitre s'adresse à l'élève B en lui demandant : "Alors, qu'est-ce que tu veux écrire ? ». Cette parole " fondatrice » en début d'atelier est déterminante pour l'atmosphère dans laquelle l'élève B va être installé pour écrire (Bucheton \& Soulé, 2009a). L’usage du verbe modal "vouloir » exprimant une modalité subjective (expression de la volonté) et le déictique «tu » permettent d'enrôler en prenant en compte la dimension psycho-affective du "sujet-écrivant » (Bucheton, 2014). En effet, le «tu » implique un « je » en retour de la part de l'élève B : «j'ai envie d'écrire il y a des mamans qui aiment le travail » (TDP 382) ; « euh, j(e) (n)’arrive pas » (TDP 384).

En terme de reformulation, au tour de parole 374, «alors, qu'est-ce que tu veux écrire ? » est une auto-répétition où l’on retrouve l'énoncé-source au tour de parole 192. Cet énoncé se retrouve de manière récurrente au début d'un épisode où le maitre apporte un étayage dans la phase de mise en texte. Ce geste langagier fonctionne pendant toute la

${ }^{18}$ Méthode phonétique et gestuelle qui associe un geste à un phonème.

${ }^{19}$ Présenté en annexe, l'extrait doit être lu dans son intégralité pour apprécier les jeux de reformulation. 
séance pour maintenir l'élève dans la tâche et le faire progresser en mettant en relation le déjà fait et les possibles de l'écriture. Au tour de parole 382, l'énoncé de l'élève $\mathrm{B}$ est une hétéro-paraphrase ayant comme énoncé-source « il y a des mamans qui » (TDP 376) et " aiment » au tour de parole d'après. Ces énoncés sont eux-mêmes des reformulations que l'on retrouve au début de la séance durant la phase de planification puisqu'elle porte sur l'écrit à produire. L'énoncé « j'ai envie d'écrire il y a des mamans qui aiment le travail » est une reformulation qui contient de l'écrit à produire (« il y a des mamans qui aiment le travail ») associé à un commentaire portant sur celui-ci : «j’ai envie d’écrire ». Celui-ci exprime une modalité subjective par l'expression de la volonté du «sujet-écrivant» (Bucheton, 2014).

En 377, l'élève B propose d'écrire « aiment » : le maitre ratifie sa proposition par une hétéro-répétition en 378 suivi d’une hétéro-paraphrase ("alors comment ça s’écrit aiment ? ») correspondant à une reformulation contenant de l'écrit à produire ( "aiment ») associé à un commentaire sur le comment-écrire ("alors comment ça s’écrit»), l'enchainement des deux reformulations permettant de faciliter le passage entre l'écrit proposé et le passage à l'écriture.

Après une courte pause, le maitre demande à un autre élève de l'aider : ce recours montre qu'il est dans une posture d'accompagnement en cherchant à réduire la dépendance de l'élève vis-à-vis de l'enseignant. L'élève a recours à un «transcodage » ${ }^{20}$ (Peytard, 1993) en épelant le début du mot («a-i-m- ») en 379 à partir de l'énoncé-source " aiment » en 377. En 380, le maitre a recours à une hétéro-répétition accompagnée d’un geste de la main (une " reformulation gestuelle " pour inciter l'élève à poursuivre ce qui entraine un effet langagier «bienheureux » puisque l'élève B et un autre élève disent « e- » en 381. Comme l'élève B est en « insécurité scripturale » (Dabène, 1991), il dit au maitre " euh $\mathrm{j}(\mathrm{e})$ (n)'arrive pas » en 384 quand celui-ci l'incite au passage à l'écriture en 383 («vas$\mathrm{y}$ »). Au lieu de lui épeler les lettres, il a encore recours à l'élève qui l'a aidé (" mais elle vient de te le dire »), ce qui montre encore la dominance de la posture d'accompagnement. L'élève $\mathrm{B}$ se lance en commençant à écrire le mot et en ayant recours à une répétition de la première lettre du mot " aiment ", l'autre élève faisant de même en épelant les deux premières lettres en 387. En 388, l'élève $\mathrm{B}$ dit au maitre "ça y est », mais le maitre a recours à une reformulation paraphrastique avec un commentaire sur l'écrit à produire : «alors, il y a plein de mamans qui aiment» (389). Ici, l'étayage porte sur le morphogramme grammatical $-n t$, la règle morphosyntaxique de l'accord en nombre du verbe étant très complexe, d'autant plus que ce morphogramme est non perceptible à l'oral (David \& Dappe, 2011). La réponse de l'élève B (s-) en 390 témoigne d'une conscience morphographique puisqu'il applique la règle de l'accord en nombre du nom et de l'adjectif. Le maitre a ensuite recours à une « méthode de raisonnement " permettant à l'élève de voir s'il faut mettre -nt par déduction puisque ce n'est ni un objet, ni une chose, ni un animal et ni une personne. Ceci permet ainsi à l'élève B de dire qu'il faut ajouter «-nt » (398). Comme nous prenons en compte les reformulations différées, la succession de questions posées à l'élève pourrait d'ailleurs être considérée comme des répétitions puisque le maitre et les élèves ont fréquemment recours à cette méthode de raisonnement.

Au tour de parole 399, le maitre relit l'écrit de l'élève B avec pointage des mots. Cette "reformulation gestuo-oralo-graphique » est fréquente chez ce maitre qui a très

\footnotetext{
${ }^{20}$ Peytard (1993) distingue 2 types d'opération dans l'altération discursive : la reformulation et le transcodage (opère d'un code à l'autre).
} 
souvent recours au pointage des mots pendant la relecture de l'écrit, Fijalkow et Fijalkow (2001) ayant montré son intérêt pour la conceptualisation de l'écrit. D’ailleurs, le maitre incite souvent l'élève $\mathrm{B}$ à relire son écrit, contrairement aux autres enseignants de notre corpus qui relisent eux-mêmes.

En 400, l'élève B initie l'échange en ayant recours à une reformulation portant sur l'écrit à produire avec un commentaire sur le comment-écrire : " comment on écrit travail maitre ». Ceci témoigne de la posture réflexive de l'élève, car il intervient spontanément sans la sollicitation du maitre. Il semble d'ailleurs très fier en disant « j'ai presque fini », ce qu'il essaye de montrer au maitre en ayant recours à une « reformulation gestuo-oralographique » (relecture avec suivi du doigt). Le maitre l'incite à continuer notamment par une hétéro-répétition ( «qui aiment le travail ») en précisant : «moi, je peux t’aider pour la fin, mais je (ne) peux pas t'aider pour le début. Tu (n’) as pas besoin de moi » (401). Le maitre fait confiance à l'élève $\mathrm{B}$ et son regard bienveillant va permettre à l'élève $\mathrm{B}$ de prendre confiance en lui en surmontant cette insécurité scripturale. Du coup, l'élève B s'exclame avec jubilation en 404 : «ça y est, j'ai écrit travail ! » (auto-reformulation paraphrastique avec " écrit dans l'oral » et commentaire sur celui-ci). Le maitre adopte ensuite une « posture d'enseignement » en nommant le savoir en jeu : il relit une partie de l'écrit en pointant du doigt le mot «travail » en 405 («reformulation gestuo-oralographique ») puis dit : "il manque juste un l- ». L'élève B relit ensuite son écrit à la demande du maitre (TDP 406), ce qui correspond à une « reformulation oralo-graphique » dans notre typologie.

Le maitre demande ensuite à l'élève $\mathrm{B}$ de lire la suite sur le TBI en pointant du doigt les mots, l'élève $\mathrm{B}$ lisant l'écrit, ce qui correspond à une auto-répétition avec continuation, l'énoncé-source étant au TDP 408 ( « reformulation gestuo-oralo-graphique » avec un commentaire sur l' " écrit dans l’oral » ) : " et des mamans qui et cetera ».

Le maitre répète à deux reprises la fin de l'écrit lu par l'élève en ajoutant « aiment » et un commentaire sur le quoi-dire, autrement dit sur la formulation de l'écrit à produire ( « qui aiment quoi ?) en 411. L'élève B propose d’écrire « jouer » en 412. Le maitre donne son accord puis il dit à l'élève $B$ : « je te laisse continuer ».

Au tour de parole 426, l'élève B initie l'échange (indice de «posture réflexive ») en demandant " comment on écrit jouer ? ", ce qui correspond à une reformulation avec de l'écrit à produire («jouer») accompagné d’un commentaire sur le comment-écrire. Le maitre répète le mot « jouer » en marquant une pause entre les deux syllabes du mot avec une accentuation sur la première syllabe, ce qui correspond à une "reformulation prosodique » pour faciliter l'encodage, mais l'élève B propose la lettre g- en 428. Au tour de parole 429, le maitre auto-répète "l'autre lettre qui chante [3] » (posture d'enseignement), ce qui permet à l'élève $\mathrm{B}$ de dire « j- » en 430 et de l'écrire. Au tour de parole 433, l'élève B répète « j- » et « JOU-er » (une auto- et une hétéro-répétition) comme une sorte de «langage intérieur oralisé ». Il initie l'échange au tour de parole 436, ce qui montre encore sa posture réflexive, en disant : «maitre c'est e-t- ou... ? ». Le maitre adopte encore une posture d'enseignement pour installer le rapport à la norme en lui répondant en 439 : « alors c'est le [e] e-r-». Dès qu'il a fini d'écrire le mot « jouer », l'élève B dit « ça y est » comme un cri de victoire. Le maitre lui demande ce qu'on met à la fin de la phrase et l'élève $\mathrm{B}$ répond « un petit point ». Puis il demande à l'élève $\mathrm{B}$ de relire son écrit en explicitant les finalités de sa demande : « il faut que je voie s'il y a bien tous les mots ». La relecture de la phrase par l'élève $B$ est une "reformulation gestuo-oralo-graphique » 
puisque le maitre pointe les mots lus par l'élève : « il y a des mamans qui aiment le travail et des mamans qui aiment jouer » (444). Au tour de parole 445, le maitre emploie ensuite un appréciatif ( « parfait »), mais ne compte pas en rester là puisqu’il demande à l’élève $B$ d'écrire une autre phrase avec un « on » inclusif ( "alors maintenant on va en écrire une deuxième ») qui a une fonction de réassurance et qui signifie : « ne t’inquiète pas, je suis là pour t'aider ». Le maitre utilise ensuite une auto-paraphrase peut-être pour expliciter le mot « écrire » qui est polysémique : « on va en inventer une deuxième ». Les deux énoncés que nous venons de citer peuvent aussi être considérés comme des répétitions puisque le maitre les utilise de manière récurrente durant la séance.

\section{Synthèse et discussion}

L'analyse linguistique de cet extrait portant sur l'étayage apporté par le maitre d'une classe très efficace à un élève " fragile » montre le rôle des reformulations dans la co-construction du savoir portant sur une phrase à écrire proposée par l'élève $\mathrm{B}$. Ces reformulations contiennent, en général, de l'écrit à produire, en cours de production ou produit. À partir de l'analyse de cet extrait, nous pouvons constater que :

- La dimension paraverbale comme la prosodie (les pauses, l'accentuation) dans «la reformulation prosodique » et la dimension non verbale comme les gestes de la main ( « reformulation gestuelle » et « reformulation gestuo-oralo-graphique ») viennent étayer la parole du maitre dans l’aide à l'encodage phonographique.

- Les «reformulations gestuo-oralo-graphiques » consistant à relire l'écrit produit avec pointage des mots sont fréquentes dans la séance 8, et notamment dans l'extrait étudié. Cette incitation à faire relire l'écrit par l'élève nous parait intéressante, celle-ci permettant de renforcer le lien lecture-écriture. De plus, le pointage du doigt renforce la conceptualisation de la langue écrite (Fijalkow \& Fijalkow, 2001).

- Les commentaires associés aux reformulations contenant de l' " écrit dans l'oral » (Marty, 1991) portent notamment sur le « comment écrire » ou le « quoi dire ». Ils permettent de développer une posture réflexive par rapport à l'écrit en favorisant le développement des compétences métascripturales (cf. les commentaires métagraphiques selon Jaffré (2003) ou David (2003)). Nous pensons aussi comme Bucheton (2009) que l'hétéro-reformulation du maitre autrement dit « la reprise fréquente du propos des élèves pour le leur renvoyer (geste-miroir) ou la reformulation commentée du propos favorise une plus grande réflexivité » (p. 64).

- Les énoncés du maitre au début de l'extrait (374. M : «Alors, qu'est-ce que tu veux écrire ? ») et à la fin de celle-ci (445. M : "Alors maintenant on va en écrire une deuxième. On va en inventer une deuxième ») sont des répétitions qui initient et closent les échanges avec chaque élève lorsque le maitre apporte un étayage individuel lors de la tâche d'écriture. Nous pouvons considérer chacun de ces épisodes comme des «scénarios » stables ou préétabli au sens de Bruner, ce qui favorise les apprentissages.

L'étude des reformulations de l'élève B permet aussi de se rendre compte que la didactique de l'écriture est une didactique intégratrice : en effet, les reformulations avec recours à l'épellation de l'élève B montrent que les élèves renforcent leur connaissance des lettres (ex. au TDP 386); les « reformulations gestuo-oralo-graphiques » permettent de renforcer les compétences en lecture (ex. au TDP 400); la «reformulation phonoprosodique » permet de segmenter les syllabes et faciliter l'encodage (ex. au TDP 433) ; les reformulations contenant de l'écrit à produire, en cours de production ou produit 
accompagnées d'un commentaire permettent d'adopter une posture réflexive par rapport à l'écrit (TDP 400) ou/et de prendre en compte la dimension psycho-affective (TDP 382).

On peut donc faire l'hypothèse que la reformulation orale est un élément de l'observatoire des pratiques littéraciées. En effet, son étude, lors d'un atelier d'écriture au $\mathrm{CP}$, permet de rendre compte de l'articulation du lire-écrire-parler et du développement de l'élève.

Cependant, l'étude des reformulations ne suffit pas à rendre compte de ce qui se joue dans les interactions. Nous avons associé à cette étude le «modèle des postures d'étayage » (Bucheton \& Soulé, 2009b) ainsi que la linguistique de l'énonciation pour trouver des traces de la subjectivité du «sujet écrivant " (Bucheton, 2014). Celui-ci est considéré par l'auteure comme « une personne singulière avec une histoire, des émotions, un engagement sensé dans ce qu'il dit ou fait et qui, pour ce faire, pense, communique avec son stylo ou son clavier » (p. 11). Nous avons montré que l'enseignant était dans une " posture d'accompagnement » dominante ce qui favorisait une " posture réflexive » de l'élève $B$. Nous faisons l'hypothèse que cette posture d'accompagnement du maitre favorise les reformulations de l'élève puisqu'elles sont nombreuses dans les échanges. L'usage du déictique «tu » du maitre et du verbe modal « vouloir » de la part du maitre (374 : « alors, qu’est-ce que tu veux écrire ? ») impliquent respectivement un « je » de la part de l'élève B ainsi que la prise en compte de la dimension psycho-affective (382 : «j’ai envie d'écrire ... »). Cette dimension parait fondamentale, car l'élève B est en insécurité scripturale : en effet, il manque de confiance en lui et n'ose pas se lancer (384 : « euh, j'arrive pas »), l'acte d'écrire pouvant être anxiogène pour certains élèves. L'étayage ainsi que l'attitude bienveillante du maitre permet à l'élève de modifier son «rapport à l'écriture ${ }^{21} \gg$ (Barré-De Miniac, 2000), ce qui prouve que la compétence scripturale ne se limite pas à des connaissances sur la langue ou à la maitrise d'une technique, le rapport à l'écriture en faisant partie (ibid.).

\section{Conclusion}

À travers l'étude de la reformulation orale, l'analyse linguistique de cet extrait de verbatim dans une des classes les plus efficaces de la recherche Lire Écrire $C P$, montre que :

- La reformulation peut être considérée comme une ressource langagière orientée pour l'action à visée didactique, c'est-à-dire pour l'articulation du lire-écrire-parler.

- L'étude de la reformulation dans un observatoire des pratiques littéraciées permet de se rendre compte que la didactique de l'écriture en première année de primaire (CP) est une didactique qui peut être intégratrice quand elle prend en compte le développement linguistique, cognitif et identitaire du sujet écrivant (Bucheton, 2014) qui inclut les compétences en littératie.

- La reformulation de l'enseignant peut être considérée comme un « geste professionnel » langagier qui joue un rôle déterminant dans l'efficacité ainsi que l'équité des pratiques et qui conditionne l’oral réflexif. L’enseignant peut être qualifié de « médiateur de littératie »

\footnotetext{
21 « désigne des conceptions, des opinions, des attitudes, de plus ou moins grande distance, de plus ou moins grande implication, mais aussi des valeurs et des sentiments attachés à l'écriture, à son apprentissage et à ses usages » (Barré-De Miniac, 2000, p.13).
} 
(mediator of literacy 22 ou literacy mediator), si l'on reprend et traduit un concept issu des New Literacy Studies (Baynham, 1995 ; Papen, 2010), et l'étayage consistant à s'ajuster en permanence pour accompagner l'élève lors d'un atelier d'écriture nous semble être un indice d'expertise professionnelle.

Dans le cadre de cet article, nous avons considéré la reformulation orale comme un médium d'enseignement/apprentissage. À l'instar de Lafontaine et Hébert (2015), GarciaDebanc (2007) et Noyau (2010), nous faisons l'hypothèse qu'elle peut aussi être un objet d'enseignement en formation (Castany-Owhadi, 2015).

\section{Références}

Apothéloz, D. (2005). Progression du texte dans les rédactions conversationnelles : les techniques de la reformulation dans la fabrication collaborative du texte, in: R. Bouchard \& L. Mondada (Eds.), Les processus de la rédaction collaborative (pp. 165199). Paris : L’Harmattan.

Apothéloz, D. (2007). Note sur l'activité de reformulation dans la conversation. Recherches linguistiques, 29, 145-162.

Barré-De Miniac, C. (2000). Le rapport à l'écriture. Villeneuve d'Ascq: Presses universitaires du Septentrion.

Baynham, M. (1995). Literacy practices : Investigating literacy in social contexts. London and New York : Longman.

Besse, J.-M. (2000). Regarde comme j'écris : écrits d'élèves, regards d'enseignants. Paris : Magnard.

Brixhe, D., \& Spécogna, A. (1999). Actes de reformulation et progression du savoir. Pratiques, 103-104, 9-27.

Bruner, J. (1983). Le développement de l'enfant : savoir faire, savoir dire. Paris : Presses Universitaires de France.

Bucheton, D. (2009). L'agir enseignant : des gestes professionnels ajustés. Toulouse : Octarès Éditions.

Bucheton, D., \& Soulé, Y. (2009a). L’atelier dirigé d'écriture au CP : une réponse à l’hétérogénéité des élèves. Paris : Delagrave.

Bucheton, D., \& Soulé, Y. (2009b). Les gestes professionnels et le jeu des postures de l'enseignant dans la classe : un multi-agenda de préoccupations enchâssées. Éducation \& Didactique, 3(3), 29-48.

Bucheton, D. (2014). Refonder l'enseignement de l'écriture. Vers des gestes professionnels plus ajustés du primaire au lycée. Paris : Retz.

Castany-Owhadi, H. (2015). La reformulation de l'enseignant comme objet d'étude en formation? Construction dialoguée de récit en maternelle. Communication à la journée d'étude «Gestes langagiers et gestes professionnels dans les activités de lecture et d'écriture », Montpellier, 30 janvier 2015.

Castany-Owhadi, H., Soulé, Y., \& Dreyfus, M. (soumis). La reformulation orale au sein de l'atelier d'écriture au Cours Préparatoire: Analyse descriptive et comparative dans une visée compréhensive de l'activité de l'enseignant pour l'apprentissage de l'écriture. Namur : Presses universitaires de Namur, collection Diptyque.

22 « can be defined as a person who makes his or her literacy skills available to others, on a formal or informal basis, for them to accomplish specific literacy purposes » (Baynham, 1995, p.39). 
Chabanne, J.-C., \& Bucheton, D. (2002). Parler et écrire pour penser apprendre et se construire : L'écrit et l'oral réflexifs. Paris : PUF.

Chemla, M.-T., \& Dreyfus, M. (2002). L’oral « intermédiaire » dans la lecture littéraire en cycle 2. Étude d'un moment de lecture-feuilleton en GS : Samani l'indien solitaire, in : J.-C. Chabanne \& D. Bucheton (Eds.), Parler et écrire pour penser, apprendre et se construire : L’écrit et l'oral réflexifs (pp. 99-121). Paris : PUF.

Chomsky, C. (1971). Write first, read later. Childhood Education, 47, 296-299.

Dabène, M. (1991). Un modèle didactique de la compétence scripturale. Repères, 3, 25-44.

David, J. (2003). Linguistique génétique et acquisition de l'écriture. Faits de langue, 22, 37- 45.

David, J., \& Dappe, L. (2011). Interactions et morphologie grammaticale écrite à l'école primaire. Recherches, 54, 17-31.

De Gaulmyn, M.-M. (1987). Actes de reformulation et processus de reformulation, in : P. Bange (Eds.), L'analyse des interactions verbales. La Dame de Caluire. Une consultation (pp. 83-98). Berne : P. Lang.

Dreyfus, M., Soulé, Y., Dupuy, C., \& Castany-Owhadi, H. (2017). Les tâches d’écriture au CP : des pistes pour la formation ? Repères, 55, 45-64.

Ehri, L. C. (1989). Movement into word reading and spelling - How spelling contributes to reading, in : J. M. Mason (Eds.), Reading and Writing Connections. Boston : MA, Allyn and Bacon.

Ferreiro, É., \& Gómez Palacio, M. (1988). Lire-écrire à l’école : comment s’y apprennentils? Analyse des perturbations dans les processus d'apprentissage de la lecture et de l'écriture. Lyon : CRDP.

Fijalkow, É., \& Fijalkow, J. (2001). La lecture accompagnée d'un suivi du doigt : étude exploratoire, in : G. Chauveau (Eds.), Comprendre l'enfant apprenti lecteur (pp. 159181). Paris : Retz.

François, F. (1990). La communication inégale. Heurs et malheurs de l'interaction verbale. Paris : Delachaux et Niestlé.

Gagné, G., Lazure, R., Sprenger-Charolles, L., Ropé, F. et al. (1989). Recherches en didactique et acquisition du français langue maternelle, (Tome 1 - Cadre conceptuel, thésaurus et lexique des mots-clés ; Tome 2 - Répertoire bibliographique). BruxellesParis-Montréal : De Boeck Université.

Garcia-Debanc, C. (2003). Faire écrire les élèves dès le début du CP, in : B. Germain, I. le Guay \& N. Robert (Eds.), Le manuel de lecture au CP. Réflexions, analyses et critères de choix (pp. 67-77). Paris : Savoir Livre / CNDP.

Garcia-Debanc, C. (2007). La reformulation orale: un élément de l'expertise professionnelle, in : L. Talbot \& M. Bru (Eds.), Des compétences pour enseigner. Entre objets sociaux et objets de recherche (pp. 151-168). Rennes : PUR.

Garcia-Debanc, C. (2012). Synthèse sur la journée d'étude « La reformulation : usages et contextes ». Poitiers: Université de Poitiers. Récupéré du site $<$ http://uptvpoitiers.fr/program/la-reformulation-usagesetcontextes/video/3470/synthese-de-la-journee-d-etude/index.html>.

Goigoux, R., Jarlégan, A., \& Piquée, C. (2015). Évaluer l’influence des pratiques d'enseignement du lire-écrire sur les apprentissages des élèves. Recherches en didactiques, 19, 33-52. 
Goigoux, R. (2016). Lire et Écrire, étude de l'influence des pratiques d'enseignement de la lecture et de l'écriture sur la qualité des premiers apprentissages. Récupéré du site de l’Institut français de l'éducation (IFE) : <http://ife.ens-lyon.fr/ife/recherche/lireecrire>.

Goody, J. (1987). Entre l’oralité et l'écriture. Paris : PUF.

Gülich, E., \& Kotschi, T. (1987). Les actes de reformulations dans la consultation le dame de Caluire, in : P. Bange (Eds.), L’analyse des interactions verbales. La dame de Caluire : une consultation (pp. 15-81). Berne : Peter Lang.

Hébert, M. (2010). Comment aider TOUS les élèves à mieux apprécier un roman, in : M. Hébert \& L. Lafontaine (Eds.), Littératie et inclusion (pp. 151-178). Québec : Presses de l’Université du Québec.

Hébert, M., \& Lafontaine, L. (2010). Littératie et école inclusive. Un double défi pour l'école d'aujourd'hui, in : M. Hébert et L. Lafontaine (Eds.), Littératie et inclusion (pp. 1-10). Québec : Presses de l’Université du Québec.

Hébert, M., \& Lépine, M. (2013). De l'intérêt de la notion de littératie en francophonie : un état des lieux en sciences de l'éducation. Globe, 161, 25-43.

Hudelot, C. (1993). Du noviciat de l'expert. Étayage, débrayage et cafouillages dans un dialogue adulte enfant. Cahiers de linguistique sociale, 23, 51-83.

Jaffré, J.-P. (2003). Les commentaires métagraphiques. Faits de langue, 22, 67-76.

Jaubert, M., \& Rebière, M. (2001). Pratiques de reformulation et construction de savoirs. ASTER, 33, 81-110.

Jaubert, M., \& Rebière, M. (2002). Parler et débattre pour apprendre : comment caractériser un « oral réflexif », in : J.-C. Chabanne \& D. Bucheton (Eds.), Parler et écrire pour penser, apprendre et se construire : L’écrit et l’oral réflexifs (pp. 163-186). Paris : PUF.

Lafontaine, L., \& Hébert, M. (2015). La reformulation comme outil langagier pour justifier son opinion dans les cercles de lecture entre pairs : effets d'un enseignement de l'oral, in : R. Bergeron, C. Dumais, B. Harvey \& R. Nolin (Eds.), La didactique du français oral au Québec (pp. 119-140). Québec : Les presses de l’Université du Québec.

Lahire, B. (2008). La raison scolaire. École et pratiques d'écriture, entre savoir et pouvoir. Rennes : PUR.

Marin, B. (2011). La reformulation en classe : un discours équivoque, in : J.-Y. Rochex \& J. Crinon (Eds.), La construction des inégalités scolaires. Au cœur des pratiques et des dispositifs d'enseignement (pp. 77-88). Rennes : PUR.

Marty, N. (1991). Genèse de l'écrit et activités métalinguistiques dans le dialogue de jeunes scripteurs. Études de Linguistique Appliquée, 81, 57-70.

Mauroux, F., David, J., \& Garcia-Debanc, C. (2015). Analyse des actions et des interactions didactiques en production écrite au Cours Préparatoire. Repères, 52, 121141.

Milian, M. (2005). Reformulation : a Means of Constructing Knowledge in Shared Writing. Educational Studies in Language and Literature, 5(3), 335-351.

Moreau, A. C., Leclerc, M., \& Landry, L. (2010). Les ateliers de lecture et d'écriture au préscolaire: expériences d'enseignement-apprentissage, in : M. Hébert \& L. Lafontaine (Eds.), Littératie et inclusion (pp. 37-57). Québec : Presses de l'Université du Québec. 
Morin, M.-F., \& Montésinos-Gelet, I. (2007). Effet d'un programme d'orthographes approchées en maternelle sur les performances ultérieures en lecture et en écriture d'élèves à risque. Revue des sciences de l'éducation, XXXIII(3), 663-683.

Moussi, D. (2015). Re-formuler... ou comment prendre en compte les réponses des élèves pour avancer dans l'interaction ? Recherches, 62, 51-71.

Nonnon, É. (1990). Est-ce qu'on apprend en discutant ? Interaction maitre-élèves en S.E.S., in : F. François (Eds.), La communication inégale. Heurs et malheurs de l'interaction verbale (pp. 147-212). Paris : Delachaux et Niestlé.

Noyau, C. (2010). Développer les capacités de reformulation chez les maîtres de l'école de base en contexte subsaharien ?. Congrès Mondial de Linguistique Française -CMLF 2010, France, p.553-571.

Papen, U. (2010). Literacy mediators, scribes or brokers? The central role of others in accomplishing reading and writing. Langage et société, 133, 63-82.

Peytard, J. (1993). D'une sémiotique de l'altération. Revue de sémio-linguistique des textes et discours, 8, 137-169.

Rabatel, A. (2010). Les reformulations pluri-sémiotiques en contexte de formation. Besançon : Presses universitaires de Franche-Comté.

Rieben, L. (2003). Écritures inventées et apprentissage de la lecture et de l'orthographe. Faits de langue, 22, 27-36.

Rieben, L., Ntamakiliro, L., Gonthier, B., \& Fayol, M. (2005). Effects of various early writing practices on reading and spelling. Scientific Studies of Reading, 9, 145-166.

Sénéchal, M., Ouellette, G., Pagan, S., \& Lever, R. (2012). The role of invented spelling on learning to read in low-phoneme-awareness kindergartners: A randomizedcontrol-trial study. Reading and Writing, 25(4), 917-934.

Sensevy, G. (2011). Le sens du savoir. Éléments pour une théorie de l'action conjointe en didactique. Bruxelles : De Boeck.

Totereau, C., \& Dreyfus, M. (dir.) (2017). Littéracie et entrée dans l'écrit. Compétences des élèves et pratiques enseignantes. Lidil, 55.

Volteau, S., \& Garcia-Debanc, C. (2008). Gérer les reformulations : un geste professionnel. Influence des objets enseignés sur les types de reformulations, in : D. Bucheton \& $\mathrm{O}$. Dezutter (Eds.), Le développement des gestes professionnels dans l'enseignement du français. Un défi pour la recherche et la formation (pp. 191-212). Bruxelles : De Boeck.

\section{Biographies des auteurs}

Hélène Castany-Owhadi, enseignante spécialisée chargée de l'aide aux élèves en difficulté, est doctorante en Sciences du Langage à l'Université de Montpellier. Sous la direction de Martine Dreyfus et d'Yves Soulé, elle travaille sur la reformulation orale dans les situations d’enseignement-apprentissage de la lecture et de l'écriture.

Yves Soulé est maitre de conférences en Sciences du langage à l'université de Montpellier. Ses recherches concernent la didactique du français - lecture et littérature. Il a participé à l'élaboration du modèle des gestes professionnels et s'intéresse à la dimension langagière de la professionnalité enseignante. 
Martine Dreyfus est Professeure émérite en Sciences du langage à l’Université de Montpellier. Ses travaux de recherche actuels portent sur l'analyse des pratiques langagières dans l'enseignement/apprentissage du français, langue maternelle ou première, sur la relation entre gestes langagiers et gestes professionnels, sur les pratiques enseignantes dans le domaine de l'écriture et de la lecture au début de l'école primaire.

Annexe 1

Extrait : «Il y a des mamans qui aiment le travail et des mamans qui aiment jouer »

\begin{tabular}{|c|c|}
\hline $\begin{array}{l}\text { 374. M : (...) qu'est'-ce que tu } \\
\text { veux écrire'+/ }\end{array}$ & -auto-répétition (ES 23192$)$ \\
\hline \multicolumn{2}{|l|}{ 375. B : XXXX } \\
\hline 376. $M$ : il y a des mamans qui+ & $\begin{array}{l}\text {-hétéro-répétition avec continuation (ES16); } \\
\text { (« écrit dans l’oral ») }\end{array}$ \\
\hline \multicolumn{2}{|l|}{ 377. B : aiment } \\
\hline $\begin{array}{l}378 . \quad \mathrm{M} \quad \text { : aiment, }+/ \text { alors } \\
\text { comment' ça s'écrit aiment,+/ (à } \\
\text { l'élève qui est en face de B) tu peux } \\
\text { l'aider'+/ }\end{array}$ & $\begin{array}{l}\text {-hétéro-répétition (ES377) ( écrit dans l'oral ») } \\
\text {-hétéro-reformulation paraphrastique (ES377) } \\
\text { ( écrit dans l'oral ») ; reformulation avec un } \\
\text { commentaire sur l' "écrit dans l'oral ». }\end{array}$ \\
\hline \multicolumn{2}{|l|}{ 379. E : oui,+/ $a-i-m-,+/$} \\
\hline $\begin{array}{l}\text { 380.M : } \boldsymbol{a}-\boldsymbol{i}-\boldsymbol{m} \text { (gesticulation de la } \\
\text { main) }\end{array}$ & $\begin{array}{l}\text {-hétéro-répétition (ES379) ( « écrit dans } \\
\text { l’oral »); reformulation gestuelle }\end{array}$ \\
\hline \multirow{2}{*}{$\begin{array}{l}\text { 381. E et B : e-,+/ } \\
\text { 382. B : j'ai envie d'écrire il y a } \\
\text { des mamans qui aiment le } \\
\text { travail,+/ }\end{array}$} & \\
\hline & $\begin{array}{l}\text {-hétéro- et auto-reformulation paraphrastique } \\
\text { (ES376+377) (« écrit dans l'oral »); } \\
\text { reformulation avec un commentaire sur l' « écrit } \\
\text { dans l'oral ». }\end{array}$ \\
\hline \multicolumn{2}{|l|}{ 383. $\mathrm{M}:$ vas-y,+/ } \\
\hline 384. B : euh+ j(e) (n’)arrive pas,+/ & \\
\hline
\end{tabular}

${ }^{23}$ Énoncé-source 


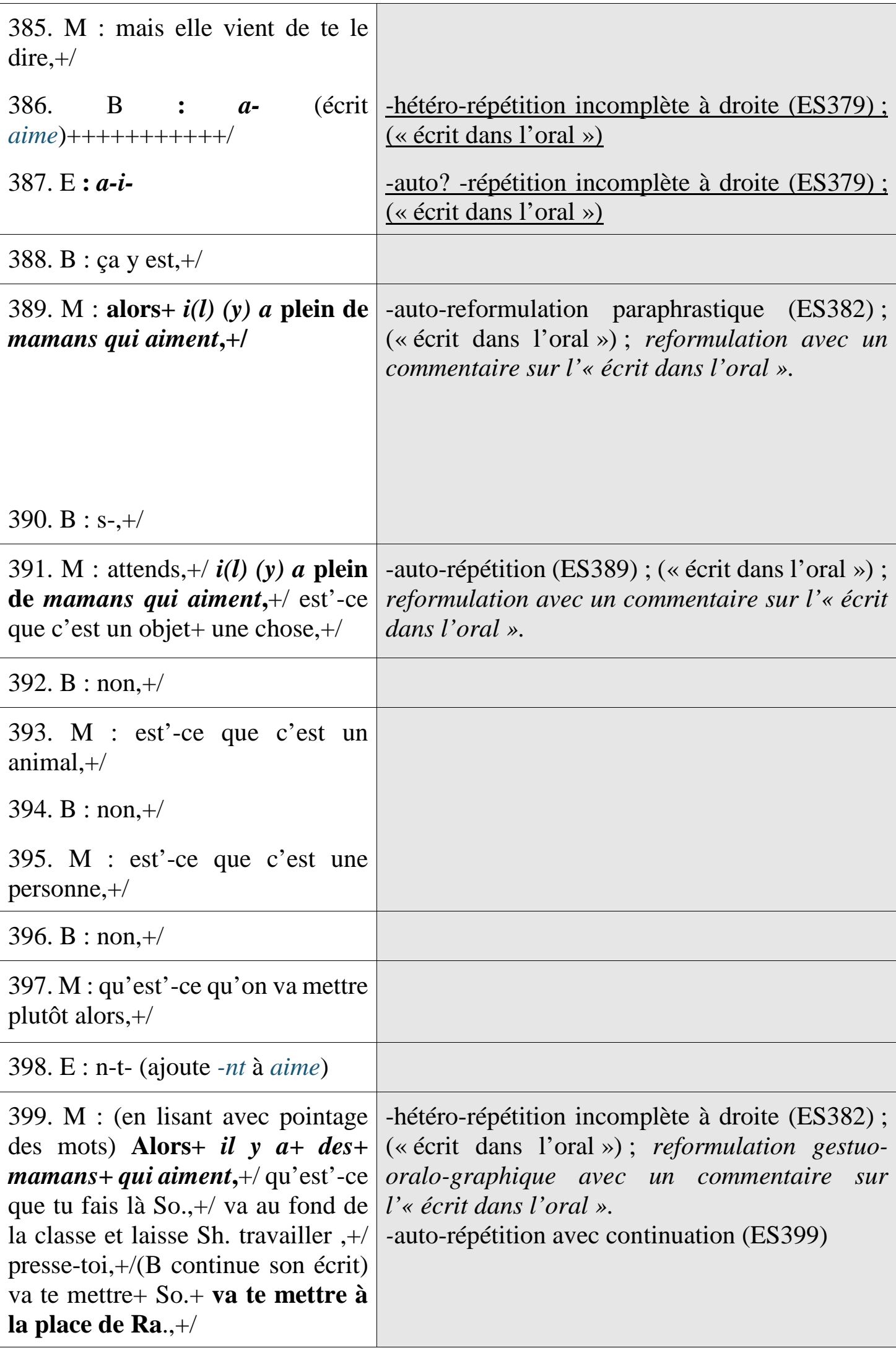


400. B : comment' on écrit travail maitre, $+/$ j'ai presque fini, $+/$ (en lisant avec suivi du crayon) a+des + mamans + qui aiment le (en oralisant) travail,+l

401. M : ben vas-y+ je t'écoute moi, $++++++/$ qui aiment le travail,++++/ moi je peux t'aider pour la fin, mais je (ne) peux pas t'aider pour le début, $+/$ tu (n)'as pas besoin de moi, ++++++++ (B écrit travai) /

402. E : là je (ne) peux pas mettre $\mathrm{XX}+\mathrm{il}$ est déjà dans $\mathrm{XXX},+$ /

403. $\mathrm{M}$ : chut + / je sais ma pauvre $\mathrm{XXXX},+1$

404. B : (a écrit travai ) ça y est+ j'ai écrit travail,+/

405. $\mathrm{M}$ : (à une autre élève) tu te forces,+/ (à B) ALORS,+/ c'est presque ça, $+/$ non je vais t'aider pour la fin,+/ d'accord'+/ (en relisant tout en pointant du doigt le travai ) $\mathbf{l e}+$ travail, $+/$ il manque juste un $1-,+++++++(B$ ajoute un $l-$ ) / alors t(u) (n')as pas fini,+/ regarde, + / relis-moi le début, + (avec geste de pointage)/

406. B : (en lisant) il+y+a+des+ mamans + qui + aiment + le+ travail,+/

407. $\mathrm{M}$ : et on continue, (le maitre se dirige vers le tableau numérique en pointant la suite de l'écrit) +/ lis, + +

408. B : et -auto-reformulation paraphrastique (ES382); (" écrit dans l'oral »); reformulation avec un commentaire sur l'« écrit dans l'oral ». -auto-répétition (ES382); ( écrit dans l'oral »); reformulation gestuo-oralo-graphique

-hétéro-répétition (ES382); (« écrit dans l'oral »)

-auto-reformulation paraphrastique (ES382); (" écrit dans l'oral ») ; reformulation avec un commentaire sur l'« écrit dans l'oral ».

-hétéro-répétition (ES382); (« écrit dans l'oral ») ; reformulation gestuo-oralo-graphique

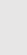

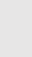

-auto-répétition (ES382); ( écrit dans l’oral ») reformulation oralo-graphique 


\begin{tabular}{|c|c|}
\hline $\begin{array}{l}\text { 409. } \mathrm{M} \text { : attends+ (a)ttends, }+/ \text { lis } \\
\text { d'abord,+/ }\end{array}$ & -auto-répétition (ES409) \\
\hline $\begin{array}{l}\text { 410. B : (en lisant avec suivi du } \\
\text { doigt du maitre) et } \text { des }^{+} \\
\text {mamans+ qui+ etcetera }+ \text { +/ }\end{array}$ & $\begin{array}{l}\text {-auto-répétition avec continuation (ES89); } \\
\text { « écrit dans l'oral »); reformulation gestuo- } \\
\text { oralo-graphique avec un commentaire sur } \\
\text { l'«écrit dans l'oral». } \\
\end{array}$ \\
\hline $\begin{array}{l}\text { 411. M : qui aiment } \\
\text { quoi’+/ qui aiment quoi’+/ }\end{array}$ & $\begin{array}{l}\text {-hétéro-reformulation paraphrastique (ES410); } \\
\text { (« écrit dans l'oral ») ; reformulation avec un } \\
\text { commentaire sur l'« écrit dans l'oral ». } \\
\text {-auto-répétition (ES411); (" écrit dans l'oral ») } \\
\text { reformulation avec un commentaire sur l'« écrit } \\
\text { dans l'oral». }\end{array}$ \\
\hline \multicolumn{2}{|l|}{ 412. B : j::::: jouer, + / } \\
\hline $\begin{array}{l}\text { 413. } \mathrm{M} \text { : d'accord,+/ (en pointant } \\
\text { du doigt sur une ligne) alors tu vas } \\
\text { continuer ici+ tu as de la place } \\
\text { encore, }+ \text { / je te laisse continuer, }+ \text { / }\end{array}$ & -auto-reformulation paraphrastique (ES413) \\
\hline $\begin{array}{l}\text { 414. } E: \quad \mathrm{j}(\mathrm{e})(\mathrm{n}) \text { ai plus de } \\
\text { place, }{ }^{+/} \text {maitre, }+/ \mathbf{j ( e ) ( \mathbf { n } ^ { \prime } ) \text { ai plus }} \\
\text { de place, }+/\end{array}$ & -auto-répétition (ES414) \\
\hline \multicolumn{2}{|l|}{$\begin{array}{l}\text { 415. } M \text { : ben à la ligne, }+/ \text { très } \\
\text { bien, }+ \text { je vais venir te voir après } \\
\text { Ba., }++++++++/\end{array}$} \\
\hline 416. E : des+ mamans,+/ & $\begin{array}{l}\text {-hétéro?-répétition (ES90); ( écrit dans } \\
\text { l’oral ») }\end{array}$ \\
\hline $\begin{array}{l}\text { 417. } \mathrm{M} \text { : (à Ha.) viens Ha., }+ \text { / } \\
\text { viens vite me montrer ce } \\
\text { mot, }+++++/ \text { quel mot' tu n'arrives } \\
\text { pas à lire, }++++ \text { (Ha. montre } \\
\text { cochon) }\end{array}$ & -auto-répétition avec continuation (ES417) \\
\hline \multicolumn{2}{|l|}{ 418. un autre $\mathrm{E}:$ cochon,+/ } \\
\hline 419. $\mathrm{M}:$ cochon,,$+++++++++++/$ & -hétéro-répétition (ES418) \\
\hline
\end{tabular}




\begin{tabular}{|c|c|}
\hline $\begin{array}{l}\text { 420. E7 : (en lisant « Il y a des } \\
\text { mamans qui ») avec pointage des } \\
\text { mots par le maitre) } \mathbf{i l +} \boldsymbol{y}^{+} \boldsymbol{a}+\text { des }^{+} \\
\text {mamans }+ \\
\text { qui }++++++++++++++++++++ \\
++++(\text { en oralisant) aiment }++ \\
\text { leurs } \text { enfants,+/ }\end{array}$ & $\begin{array}{l}\text {-hétéro-répétition avec substitution (ES30 + } \\
\frac{106) ;(\text { écrit dans l’oral »); reformulation }}{\text { gestuo-oralo-graphique }}\end{array}$ \\
\hline $\begin{array}{l}\text { 421. M : c’est déjà dans l'histoire } \\
\text { ça non un peu,+/ bon+ vas-y,+/ } \\
\text { XX commence,+/ il y a des } \\
\text { mamans qui aiment leurs } \\
\text { enfants+ ET }\end{array}$ & $\begin{array}{l}\text {-hétéro-répétition avec continuation (ES420); } \\
\text { (« écrit dans l’oral ») ; reformulation } \\
\text { prosodique }\end{array}$ \\
\hline 422. E7 : et des mamans qui & $\begin{array}{l}\text {-hétéro-répétition avec continuation (ES421); } \\
\text { (« écrit dans l’oral ») }\end{array}$ \\
\hline $\begin{array}{l}\text { 423. } \mathrm{M} \text { : qui aiment qui alors, }+ \text { / } \\
\text { qui aiment qui, }+/ \mathrm{i}(\mathrm{l})(\mathrm{y}) \text { en a qui } \\
\text { aiment leurs enfants et } \mathrm{i}(\mathrm{l})(\mathrm{y}) \text { en a } \\
\text { d'autres qui aiment qui'+/ }\end{array}$ & $\begin{array}{l}\text {-hétéro-répétition avec substitution (ES420) ; } \\
\text { (« écrit dans l'oral ») ; reformulation avec un } \\
\text { commentaire sur l'" écrit dans l'oral ». } \\
\text {-auto-répétition (ES423) ; (" écrit dans } \\
\text { l’oral ») ; reformulation avec un commentaire } \\
\text { sur l'« écrit dans l'oral ». }\end{array}$ \\
\hline 424. E7 : qui aiment leur papa,+/ & $\begin{array}{l}\text {-hétéro-répétition avec substitution (ES423); } \\
\text { (« écrit dans l’oral ») }\end{array}$ \\
\hline $\begin{array}{l}\text { 425. M : leurs parents, + / allez } \\
\text { vas-y,+/ (en lisant avec suivi du } \\
\text { doigt) il y a des mamans qui (en } \\
\text { oralisant avec suivi du doigt sur la } \\
\text { ligne) aiment leurs enfants ET } \\
\text { des mamans qui aiment leurs } \\
\text { parents,+ (l'élève écrit) / }\end{array}$ & $\begin{array}{l}\text {-hétéro-correction (ES424) ; (« écrit dans } \\
\text { l’oral ») } \\
\text {-hétéro- et auto- répétition synthèse } \\
\text { (ES420+422+424+425); ( écrit dans l’oral ») ; } \\
\text { reformulation gestuo-oralo-graphique et } \\
\text { prosodique }\end{array}$ \\
\hline $\begin{array}{l}\text { 426. B : comment on écrit } \\
\text { jouer'+/ }\end{array}$ & $\begin{array}{l}\text {-auto-reformulation paraphrastique (ES412); } \\
\text { (« écrit dans l'oral »); reformulation avec un } \\
\text { commentaire sur l'« écrit dans l'oral ». }\end{array}$ \\
\hline 427. M : JOU+er & $\begin{array}{l}\text {-hétéro-répétition (ES426) ; (« écrit dans } \\
\text { l’oral ») ; reformulation prosodique }\end{array}$ \\
\hline 428. B : un g-,+/ & \\
\hline
\end{tabular}




\begin{tabular}{|c|c|}
\hline $\begin{array}{l}\text { 429. M : hum+ hum+ l'autre } \\
\text { lettre+ l'autre lettre qui chante } \\
\text { [3],+/ l'autre lettre qui chante } \\
\text { [3],+/ }\end{array}$ & $\begin{array}{l}\text {-auto-répétition avec continuation (ES429) } \\
\text {-auto-répétition (ES429) }\end{array}$ \\
\hline \multicolumn{2}{|l|}{ 430. B : j-,+ (B écrit j-) / } \\
\hline \multicolumn{2}{|l|}{$\begin{array}{l}\text { 431. M : (à une autre élève) tu t(e) } \\
\text { rappelles’ ce qu’on a dit à Wa.,+/ }\end{array}$} \\
\hline \multicolumn{2}{|l|}{ 432. E : $\quad \underline{\mathrm{XXXXXX},+/}$} \\
\hline 433. B : $\mathbf{j - + /}$ JOU-er,+/ & $\begin{array}{l}\text {-auto-répétition (ES430); (« écrit dans l’oral ») } \\
\text {-hétéro-répétition (ES427); (« écrit dans } \\
\underline{\text { l’oral »); reformulation prosodique. }}\end{array}$ \\
\hline $\begin{array}{l}\text { 434. } \mathrm{M} \text { : il y a beaucoup de } \\
\text { mamans qui aiment (montre } \\
\text { aime),+/ qu'est'-ce qu'on met,+/ }\end{array}$ & $\begin{array}{l}\text {-auto-reformulation paraphrastique variation } \\
\text { (ES389) ; (« écrit dans l'oral »); reformulation } \\
\text { gestuelle avec un commentaire sur l'« écrit dans } \\
\text { l'oral». }\end{array}$ \\
\hline 435. E : & \\
\hline \multicolumn{2}{|l|}{ 436. B : maitre c'est le e-t- ou } \\
\hline \multicolumn{2}{|l|}{ 437. M : non,+/ } \\
\hline \multicolumn{2}{|l|}{ 438. E : je (ne) sais pas,+/ } \\
\hline \multicolumn{2}{|l|}{$\begin{array}{l}\text { 439. } \mathrm{M} \text { : alors c’est le }[\mathrm{e}]+\text { e-r-,+/ } \\
\text { non (en pointant aiment écrit une } \\
\text { première fois),+/ }\end{array}$} \\
\hline \multicolumn{2}{|l|}{ 440. B : ça y est,+/ } \\
\hline \multicolumn{2}{|l|}{$\begin{array}{l}\text { 441. M : qu'est'-ce qu’on met à la } \\
\text { fin de la phrase,+/ }\end{array}$} \\
\hline \multicolumn{2}{|l|}{$\begin{array}{l}\text { 442. B : un petit point, + (met un } \\
\text { point) / }\end{array}$} \\
\hline $\begin{array}{l}\text { 443. } \mathrm{M} \text { : tu me la relis' ta phrase } \\
\text { en entier, +/ i(l) faut que je voie } \\
\text { s’i(l) y a bien tous les mots,+/ }\end{array}$ & \\
\hline
\end{tabular}




\begin{tabular}{|l|l|}
\hline $\begin{array}{l}\text { 444. B : (en lisant «Il y a des } \\
\text { mamans qui aiment le travail et } \\
\text { des mamans qui aiment jouer » } \\
\text { avec pointage du crayon) il+ } \boldsymbol{y}+\boldsymbol{a} \\
+ \text { des+ mamans+ qui+ aiment+ } \\
\text { le+ travail+ et+ des+ mamans+ } \\
\text { qui+ aiment+ jouer,+/ }\end{array}$ & $\begin{array}{l}\text {-auto- et hétéro- répétition (ES } \\
\text { reformulation gestuo-oralo-graphique }\end{array}$ \\
\begin{tabular}{|l|l|} 
445. $\mathrm{M}$ : parfait,+/ alors \\
maintenant on va en écrire une \\
deuxième,+ (en marquant d'un \\
point le début d'une ligne) / on va \\
en inventer une deuxième,+/ \\
allez,+/
\end{tabular} & $\begin{array}{l}\text {-auto-répétition (ES274-290-296) } \\
\text { mais aussi auto-répétition (ES274-290-300) }\end{array}$ \\
\hline
\end{tabular}

Annexe 2

Conventions de transcription

\begin{tabular}{ll}
$l$ & $\begin{array}{l}\text { fin d'un énoncé } \\
+\end{array}$ \\
$\begin{array}{ll}\text { OUI, BRAvo } & \text { intonation montante après ce signe } \\
\text { intonation descendante après ce signe } \\
\text { oui: bon:: } \\
\text { précède }\end{array}$ & accentuation \\
\hline
\end{tabular}

A: Tu vas bien depuis la dernière fois

$\mathrm{B}$ :

ça va

chevauchement de paroles

(rire)

description d'actions, de gestes, de mimiques (nonverbal)

(bon)jour.

prononcé.

( ) = partie non prononcée. Ici seul jour est

$\mathrm{X}, \mathrm{XX}, \mathrm{XXX}$

mot inaudible d'une ou plusieurs syllabes

$<\ldots . .>$

séquence où la transcription ou l'interprétation est 
incertaine

en italiques

en gras

en bleu
« écrit dans l'oral »

reformulation

écrit produit par l'élève

Annexe 3

Production d'écrit de l'élève B (IFé, 2013) 


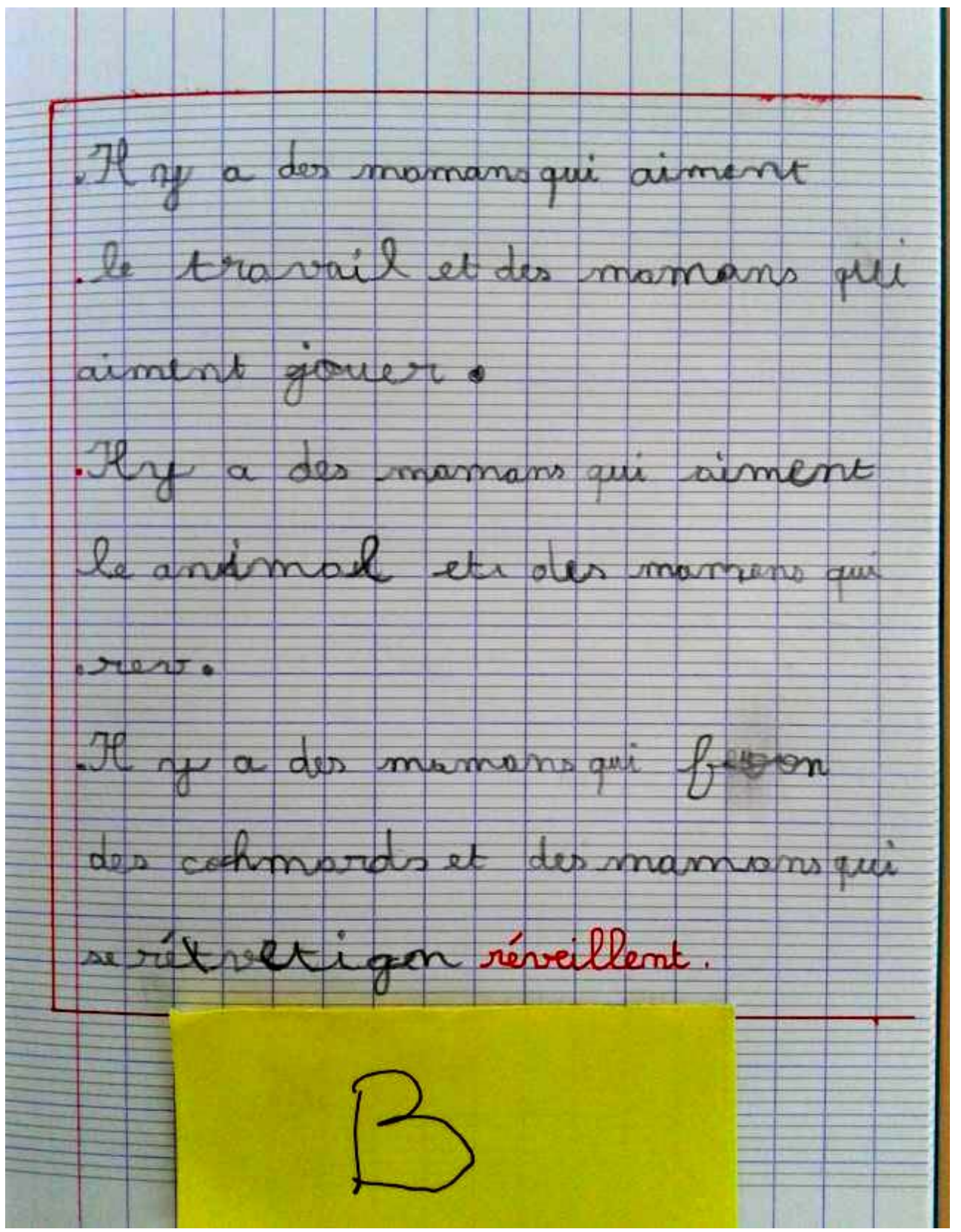

Annexe 4

Texte de l'album «Le livre des mamans » de Todd Parr (Bayard jeunesse)

\section{Le livre des mamans.}

Il y a des mamans qui conduisent des minibus, et des mamans qui conduisent des motos.

Il y a des mamans qui portent des jeans, et des mamans qui sont toujours sur leur trente et un. 
Il y a des mamans qui aiment jouer dans la neige, et des mamans qui aiment jouer sous la pluie.

Mais toutes les mamans adorent s'amuser avec leur enfant !

Il y a des mamans qui aiment danser, et des mamans qui aiment nager.

Il y a des mamans qui travaillent à la maison et des mamans qui travaillent dans des bureaux. Il y a des mamans qui sont artistes, et des mamans qui savent jouer au foot.

Mais toutes les mamans adorent regarder leur enfant dormir !

Il y a des mamans qui ont les cheveux courts et des mamans qui ont les cheveux longs.

Il y a des mamans qui aiment cuisiner, et des mamans qui aiment commander des pizzas. Il y a des mamans qui vont à la pêche, et des mamans qui font les boutiques.

Mais toutes les mamans adorent embrasser leur enfant et le prendre dans leurs bras!

Il y a des mamans qui pilotent des cerfs-volants, et des mamans qui pilotent des avions.

Il y a des mamans qui chantent des chansons, et des mamans qui lisent des histoires.

Mais toutes les mamans

aiment leur enfant comme il est ! 


\section{Annexe 5 \\ Estimation de l'ampleur des effets-classes dans la recherche LireÉcrireCP}

A partir de modèles multiniveaux intégrant, au niveau 1 , les caractéristiques des élèves et, au niveau 2, l'appartenance à une classe, les statisticiennes ont élaboré la liste des 131 classes de l'échantillon, ordonnées en fonction de leur efficacité moyenne, à caractéristiques et niveau initial des élèves donnés et elles ont extrait les classes les plus efficaces de l'échantillon (voir Jarlégan, Piquée et Fontanieu, 2016, p.329 et suiv. dans le rapport LireÉcrireCP). 